\title{
Tourism Demand Nowcasting Using a LASSO-MIDAS Model
}

\author{
Han Liu and Ying Liu \\ Center for Quantitative Economics and Business School, Jilin University \\ Changchun, 130012, PR China
}

Gang Li

School of Hospitality and Tourism Management, University of Surrey

Guildford, Surrey, GU2 7XH, UK

Long Wen

School of Economics, University of Nottingham Ningbo China

Ningbo, 315100, PR China

\begin{abstract}
Purpose - This study aims to examine whether and when real-time updated online search engine data such as the daily Baidu Index can be useful for improving the accuracy of tourism demand nowcasting once monthly official statistical data, including historical visitor arrival data and macroeconomic variables, become available.

Design/methodology/approach - This study is the first attempt to employ the LASSO-MIDAS model proposed by Marsilli (2014) to field of the tourism demand forecasting to deal with the inconsistency in the frequency of data and the curse problem caused by the high dimensionality of search engine data.

Findings - The empirical results in the context of visitor arrivals in Hong Kong show that the application of a combination of daily Baidu Index data and monthly official statistical data produces more accurate nowcasting results when MIDAS-type models are employed. The effectiveness of the LASSO-MIDAS model for tourism demand nowcasting indicates that this kind of penalty-based MIDAS model is a useful option when using high-dimensional mixed frequency data.

Originality/value - This study represents the first attempt to progressively compare whether there are any differences between using daily search engine data, monthly official statistical data, and a combination of the aforementioned two types of data with different frequencies to nowcast tourism demand. The study also contributes to the tourism forecasting literature by presenting the first attempt to evaluate the applicability and effectiveness of the LASSO-MIDAS model in tourism demand nowcasting.
\end{abstract}

Keywords Tourism demand, Nowcasting, LASSO-MIDAS, Search query data

Please cite this article as follows:

Liu, H., Y. Liu, G. Li and L. Wen (2021). Tourism demand nowcasting using a LASSO-MIDAS model. International Journal of Contemporary Hospitality Management, 33(6): 1922-1949. DOI: 10.1108/IJCHM-06-2020-0589. 


\section{Introduction}

Accurate tourism demand forecasting has enormous economic value for both publicand private-sector stakeholders. Given the perishable nature of tourism demand, tourism-related businesses rely on accurate demand forecasts to provide useful management recommendations and sales strategies (Chen et al., 2017, Liu et al., 2018, Li et al., 2019). Because the release of most statistical data is usually delayed by several weeks or months, relevant agencies and researchers have begun to explore how to accurately forecast and assess the current and recent-past tourism demand situation. Predicting the current and recent past is also called "nowcasting" and belongs to the general concept of forecasting (Banbura et al., 2011).

Accurate tourism demand nowcasting can provide valuable information for relevant agencies to assess the macroeconomic situation in real time (Lourenço et al., 2020). Previous studies have mainly focused on nowcasting macroeconomic variables, such as GDP (Dahlhaus et al., 2017), inflation (Knotek and Zaman, 2017), output growth (Rambaccussing and Kwiatkowski, 2020), and unemployment rate (Soybilgen and Yazgan, 2018), to assess current and future economic conditions in real time. Some studies focus on nowcasting issues such as influenza activity (Santillana et al., 2015), off-line polls (Ceron et al., 2015), and perceived intensity of earthquakes (Avvenuti et al., 2017). However, there have been very few such attempts in the tourism demand nowcasting literature (e.g. Lourenço et al., 2020, Wen et al., 2021). This study, therefore, aims to make a useful addition to this underdeveloped strand of tourism demand forecasting research.

Tourism demand forecasting often relies on the use of secondary data. Most of the existing tourism demand forecasting studies use visitor arrivals (hereinafter, VAs) in a specific destination to measure the total tourism demand (Song and $\mathrm{Li}, 2008$ ). In terms of key explanatory variables of tourism demand, most of the past studies on tourism demand forecasting used historical data related to tourism and macroeconomic variables for prediction (Song and $\mathrm{Li}, 2008$, Wu et al., 2017, Wen et al., 2021). In recent years, online search engine data that can reflect users' interests and intentions have been increasingly used in recent tourism demand forecasting studies (e.g. Wen et al., 2021). This type of data has been acknowledged to be a potential indicator of tourism demand (e.g. Yang et al., 2014). Some studies have shown that using search engine data to nowcast or forecast tourism demand does improve the forecasting effect (e.g. Wen et al., 2021). However, few articles have studied when this type of search engine data actually leads to a gain in nowcasting accuracy and whether such data are still useful when the tourism-related and macroeconomic variables (hereinafter, official statistical data) used in traditional tourism demand forecasting are available. This study attempts to examine these issues and provide empirical evidence.

Specifically, on the basis of the study of Wen et al. (2021), one of the few articles that use daily search engine data to nowcast tourism demand, the present study uses three different data sources to nowcast tourism demand: (1) daily search engine data 
only; (2) a combination of daily search engine data and real-time updated monthly official statistical data; (3) monthly official statistical data only. The first purpose of the present study is to investigate whether daily updated search engine data are useful for improving the accuracy of tourism demand nowcasting when monthly updated official statistical data are not available. The second purpose is to examine whether daily updated search engine data are still useful for nowcasting tourism demand once real-time updated monthly official statistical data become available. Also, if search engine data are useful when exactly do such data lead to a gain in nowcasting accuracy, and to what extent do they provide useful information?

Real-time updated search engine data can be used as a powerful tool to nowcast tourism demand, but constructing an appropriate measurement model to incorporate such data for accurate tourism demand nowcasting has encountered two technical difficulties. The first barrier is dealing with the "mixed-frequency data" problem. For example, the target variable, tourism demand, is often measured by VAs, data on which are released on a monthly basis; search engine data are updated on a daily basis. Also, the process of nowcasting tourism demand needs to add real-time updated data in sequence, and so the traditional econometrical models that allow only variables with the same frequency cannot be used. Therefore, the mixed data sampling model (hereinafter, MIDAS) proposed by Ghysels et al. (2004) is widely used because it can directly use the original frequency data for modeling without losing any data information. In particular, it allows the variables to have different sampling frequencies such that the high frequency process is projected into the low frequency process with a parsimonious weighting scheme. This method allows us to explain a low frequency variable by using exogenous variables sampled at higher frequencies without resorting to any aggregation procedure.

The second obstacle is the collinearity and overfitting that may be caused by the "curse of dimensionality" when using search engine data. The curse of dimensionality means that the number of parameters to be estimated in the model may be much larger than the sample size of the time series (Nakajima and Sueishi, 2019). The methods that can reduce the dimensionality of the data with the least possible loss of information are mainly divided into two categories. The first type of approach is represented by the principal component analysis (hereinafter, PCA) and factor models, which express common components from large amounts of data for dimensionality reduction. The second method is to reduce the number of predictors using penalized regressions with shrinkage. For example, Tibshirani (1996) proposed a technique called least absolute shrinkage and selection operator (hereinafter, LASSO), which reduces the dimensionality by constraining the coefficients of unrelated explanatory variables to zero and retaining critical explanatory variables. In recent years, some studies have begun to introduce such penalty functions into the MIDAS model for modeling and apply them to macroeconomic forecasting (e.g. Marsilli, 2014, Siliverstovs, 2017, Uematsu and Tanaka, 2017, Xu et al., 2018). Applications of penalty-based MIDAS models are mainly restricted in macroeconomic forecasting and they have not been applied in the field of tourism. Therefore, to fill the gap in the tourism forecasting literature, this study aims to compare a penalty-based MIDAS 
model and a factor-based MIDAS model in terms of their applicability and usefulness in nowcasting tourism demand.

The rest of the paper is organized as follows. The model specification section describes the methodology, the parameter estimation method, and the model evaluation methods. The section on the design of the empirical analysis describes the data, the nowcasting strategy, and how to solve the problem of inconsistent data release time in this study. The empirical results section presents the study's empirical results. Finally, the last section summarizes the conclusions and limitations of the study.

\section{Literature review}

Tourism demand forecasting methods can be roughly divided into qualitative and quantitative methods. Among them, qualitative forecasting usually depends on non-economic information such as experience and insight into the tourism market, which restricts their ability for generalization (Goh, 2012). Delphi techniques and scenario-building are the most widely used techniques in qualitative models (Song et al., 2019). On the other hand, quantitative forecasting relies on secondary data (Song and $\mathrm{Li}, 2008$ ).

As summarized by Song and Li (2008), the techniques used for quantitative tourism demand forecasting are mainly divided into time series models, econometric models, and artificial intelligence (hereinafter, AI) models.

Time series models are mainly based on historical patterns of time series data to predict future trends (Wang and Chaovalitwongse, 2011). In the existing literature, VAs, tourism revenues, tourist expenditure in the destination, and tourism import and export are the main indicators used to measure tourism demand, among which VAs have always been the most popular variable (Song et al., 2019). The most commonly used models of this type are variants of the autoregressive moving average (hereinafter, ARMA) model, such as autoregressive integrated moving average (hereinafter, ARIMA) models and seasonal ARIMA (hereinafter, SARIMA) models (e.g. Wen et al., 2021).

Econometric models can analyze the causal relationship between the dependent and independent variables that should be jointly determined (Wang and Chaovalitwongse, 2011). In terms of the determinants of tourism demand (i.e., independent variables), some studies have shown that a series of economic indicators including the destination's tourism prices, tourism prices of competitive destinations, tourist income, and exchange rates are the most important factors affecting tourism demand (Song and Li, 2008). The most commonly used econometric models to analyze the causal relationship between tourism demand and its influencing factors include the autoregressive distributed lag (hereinafter, ARDL) model (e.g. Song et al., 2012), the error correction model (hereinafter, ECM) (e.g. Goh, 2012), the vector autoregressive (VAR) model (e.g. Witt et al., 2004), and the time-varying parameter (hereinafter, TVP) model (e.g. Li et al., 2006, Wu et al., 2020).

In recent years, with the rapid development of Web technology, web data 
representing the up-to-date information can be used to supplement official statistical data. Web data such as high-dimensional search engine data that can reflect user attentions and interests have been applied to tourism demand forecasting (e.g. Sun et al., 2019). The two most commonly used search engine data are the daily Baidu Index data and the weekly or monthly Google Trends data (e.g. Li et al., 2021). However, the application of high-dimensional search engine data for tourism demand forecasting has two difficulties for the used econometric models.

The first challenge is the "mixed-frequency data" problem. For example, when using daily or weekly search engine data to predict monthly VAs, a common processing method is to aggregate high-frequency data into the monthly frequency (e.g. Wen et al., 2019); there are also studies such as Li et al. (2017) converting monthly tourist volume series into weekly series through the quadratic-match average method. However, the above processing methods of aggregating or converting the original data may cause information loss. As an alternative, Bangwayo-Skeete and Skeete (2015) were the first to apply the MIDAS model, which can directly accommodate raw mixed-frequency data, to forecast tourism demand. The MIDAS model has been widely applied in the fields of macroeconomics and finance (e.g. Andreou et al., 2010) but only recently in the field of tourism demand forecasting (e.g. Wen et al., 2021).

Regarding the use of high-dimensional search engine data, forecasting based on traditional econometric models may suffer from the curse of dimensionality, because traditional econometric models such as VAR models usually cannot accommodate too many variables in order to prevent over-parameterization. In the existing literature that used high-dimensional search engine data to predict tourism demand, researchers usually select only a few search engine data to forecast tourism demand (Pan et al., 2012, Önder and Gunter, 2016) or use shrinkage methods that can reduce the dimensionality of the data in order to use more data information (e.g. Gunter and Önder, 2016). Specifically, the existing shrinkage methods used for tourism demand forecasting mainly concentrate on factor-based models. Gunter and Önder (2016) used the Bayesian factor-augmented VAR (hereinafter, BFAVAR) model to extract common factors from 10 Google Analytics website traffic indicators to reduce the dimensionality of the VAR model, and applied them to predict VAs to Vienna. They showed that the combination model BFAVAR performs better for longer horizons. To simultaneously solve the mixed-frequency data and the curse of dimensionality problems, Wen et al. (2021) first applied a generalized dynamic factor model (GDFM) model to extract common factors from a large number of daily Baidu Index data and then applied the MIDAS-SARIMA model to predict VAs from mainland China to Hong Kong.

In recent years, in addition to the above factor-type MIDAS models, some studies have begun to introduce a type of penalized regression with shrinkage into the MIDAS model for modeling and apply them to macroeconomic forecasting. Specifically, Marsilli (2014) first proposed a LASSO-MIDAS model by combining the LASSO and the MIDAS methods and applied it to forecast US quarterly GDP growth. Subsequently, Siliverstovs (2017) merged the unrestricted MIDAS model 
(hereinafter, U-MIDAS) with the LASSO-type penalized regression called the elastic net to construct the MIDASSO model and applied it to forecast GDP growth rates in Switzerland. Some studies have combined a series of penalty functions with the U-MIDAS model and used them to predict US quarterly GDP growth rates and test the effectiveness of the proposed multiple models (Uematsu and Tanaka, 2017, Xu et al., 2018). Penalty-based MIDAS models have the following advantages in comparison with factor-type MIDAS models. First, penalty-based models can perform variable selection and model estimation simultaneously. Second, penalty-based MIDAS models can pick out variables that have a significant impact on the explained variable according to different prediction situations, thereby avoiding the difficulty of explaining the importance of the estimated factors (Marsilli, 2014, Xu et al., 2018). It should be noted that this kind of penalty-based (U-)MIDAS model is mainly used in macroeconomic forecasting and has not been applied in the field of tourism so far. The empirical results of the above studies confirm the effectiveness of the penalty-based MIDAS model in predictive performance. Therefore, this study aims to test the suitability and effectiveness of the penalty-based (U-)MIDAS model in tourism demand forecasting, and compared with factor models, whether this type of model can select from a large number of explanatory variables, thereby improving the explanatory power of the model. In addition, it should be noted that the penalty-based U-MIDAS model is not suitable for the case where the frequency difference of the mixed-frequency data is large, and this type of model does not support the case of multi-frequency explanatory variables. Therefore, this study chooses the LASSO-MIDAS model proposed by Marsilli (2014) to nowcast tourism demand.

The third category of quantitative forecasting methods is AI models, which can be used to model nonlinear relationships. The dominant models of this type include the artificial neural network (hereinafter, ANN) model (e.g. Sun et al., 2019) and the support vector regression (hereinafter, SVR) model (e.g. Zhang et al., 2017). Besides, the fuzzy time series (e.g. Tsaur and Kuo, 2011), grey theory (e.g. Sun et al., 2016), and the approach of the rough set (e.g. Goh et al., 2008) have been used in tourism demand forecasting in recent years.

This study focuses on tourism demand nowcasting and attempts to analyze important factors that affect tourism demand, which will be verified by econometric models. Therefore, we mainly provide a systematic introduction to related econometric models, and the specific introduction to the other two types of models can be found in the relevant literature such as Song and Li (2008), Song et al. (2019), and Wen et al. (2021).

\section{Nowcasting model specification}

This section introduces the LASSO-MIDAS model, focusing on the aspects of model construction and parameter estimation method, and examines methods for evaluating the model's nowcasting performance. 


\subsection{LASSO-MIDAS model}

In order to understand the construction process of the LASSO-MIDAS model more clearly, this study first introduces the basic MIDAS model proposed by Ghysels et al. (2007) and Andreou et al. (2010) which allows a set of high-frequency explanatory variables to explain low-frequency variables. The general form of MIDAS can be expressed as

$$
y_{t}=\beta_{0}+\beta_{1} B\left(L^{1 / m} ; \boldsymbol{\theta}\right) x_{t}^{(m)}+\varepsilon_{t},
$$

where $y_{t}$ is a low-frequency variable; $x_{t}^{(m)}$ is a high-frequency explanatory variable observed at most $m$ times between $t$ and $t-1$, where $t=1, \ldots, T ; \beta_{0}$ and $\beta_{1}$ are the regression coefficients to be estimated, and $\varepsilon_{t}$ is the random error term. $B\left(L^{1 / m} ; \boldsymbol{\theta}\right)=\sum_{k=1}^{K} w(k ; \boldsymbol{\theta}) L^{(k-1) / m}$ is a polynomial that determines the weights given to the high-frequency variable $x_{t}^{(m)}$ in the $L^{1 / m}$ lag operator; the $L^{(k-1) / m}$ lag operator generates the value of $x_{t}^{(m)}$ lagged by $(k-1) / m$ periods, such as $L^{(k-1) / m} x_{t}^{(m)}=x_{(t-k-1) / m}^{(m)}$. Ghysels et al. (2007) developed different parameterizations of the weight function. The estimation of all MIDAS models in this study uses the two-parameter exponential Almon lag polynomial (Marsilli, 2014); the specific form can be written as

$$
w(k ; \boldsymbol{\theta})=\frac{e^{\left(\theta_{1} k+\theta_{2} k^{2}\right)}}{\sum_{k=1}^{K} e^{\left(\theta_{1} k+\theta_{2} k^{2}\right)}} .
$$

The estimate of the MIDAS model can be obtained by minimizing the residuals of (1), which can be written as

$$
[\hat{\beta}, \hat{\theta}]=\underset{\beta, \theta}{\arg \min } \sum_{t}\left(y_{t}-\beta_{0}-\beta_{1} B\left(L^{1 / m} ; \boldsymbol{\theta}\right) x_{t}^{(m)}\right)^{2} .
$$

LASSO is a dimensionality reduction technique proposed by Tibshirani (1996). The model uses specifying properties of the $l_{1}$-norm to constrain the non-informative element $\beta_{i}$ to zero while retaining the crucial variables to reduce the dimensions of the explanatory variables. The LASSO-MIDAS model is constructed by introducing the penalty variable selection method LASSO into the MIDAS model, which can be expressed as

$$
[\hat{\beta}, \hat{\theta}]=\underset{\beta, \theta}{\arg \min } \sum_{t}\left(y_{t}-\beta_{0}-\sum_{i=1}^{n} \beta_{i} B_{i}\left(L^{1 / m} ; \boldsymbol{\theta}\right) x_{t, i}^{(m)}\right)^{2}+\lambda \sum_{i=1}^{n}\left|\beta_{i}\right|,
$$

where $\lambda$ is the regularization parameter that controls the strength of the penalty and variable selection.

The purpose of this study is to assess whether and when real-time updated high-frequency search engine data and newly released related official macroeconomic data can be useful in improving the accuracy of nowcasting tourism demand. Therefore, this study assumes that there are two kinds of data available: (1) daily-frequency search engine data and (2) monthly-frequency official statistical data, including tourism-related and macroeconomic variables. The more specific target function of the LASSO-MIDAS model can be written as follows:

$$
\min _{\beta_{0}, \beta, \theta} \sum_{t}\left(V A_{t}^{M}-\beta_{0}-\sum_{i=1}^{n_{D}} \beta_{i}^{D} B_{i}^{D}\left(L^{1 / m} ; \boldsymbol{\theta}\right) x_{t, i}^{D}-\sum_{i=1}^{n_{M}} \beta_{i}^{M} B_{i}^{M}(L ; \boldsymbol{\theta}) x_{t, i}^{M}\right)^{2}+\lambda \sum_{i=1}^{n}\left|\beta_{i}\right|,
$$

where $x_{t, i}^{D}$ is $i$-th daily Baidu Index data and $i=1, \ldots, n_{D} \cdot x_{t, i}^{M}$ is $i$-th monthly official statistical data, $i=1, \ldots, n_{M}$.

In addition, the parameter estimation method used in the LASSO-MIDAS model 
is the local regularization technique proposed by Nesterov (2005). Due to space limitations, the specific details of this method are not provided in this paper; the details can be found in Marsilli (2014).

\subsection{Selection of the regularization parameter}

The LASSO-MIDAS model involves a significant parameter selection problem: how to determine the size of the regularization parameter $\lambda$ in the model? The value of the regularization parameter $\lambda$ completely affects the nowcasting accuracy and interpretability of the LASSO-MIDAS model. The lambda value represents the strength of the regularization and determines the number of variables selected. A larger value of $\lambda$ leads to a higher intensity of regularization and fewer remaining variables (Xu et al., 2018).

Methods including conceptual predictive (hereinafter, $C_{p}$ ) statistic, cross-validation (hereinafter, CV), generalized CV (hereinafter, GCV), the Akaike information criterion (hereinafter, AIC), the Bayesian information criterion (hereinafter, BIC), and time-series CV (hereinafter, TSCV) can be used to determine the optimal value of $\lambda$. The empirical analysis in this paper uses time-series data, so the TSCV method proposed by Hyndman and Athanasopoulos (2018) is more suitable for determining the size of $\lambda$ in this paper. The specific steps are shown in Figure 1.

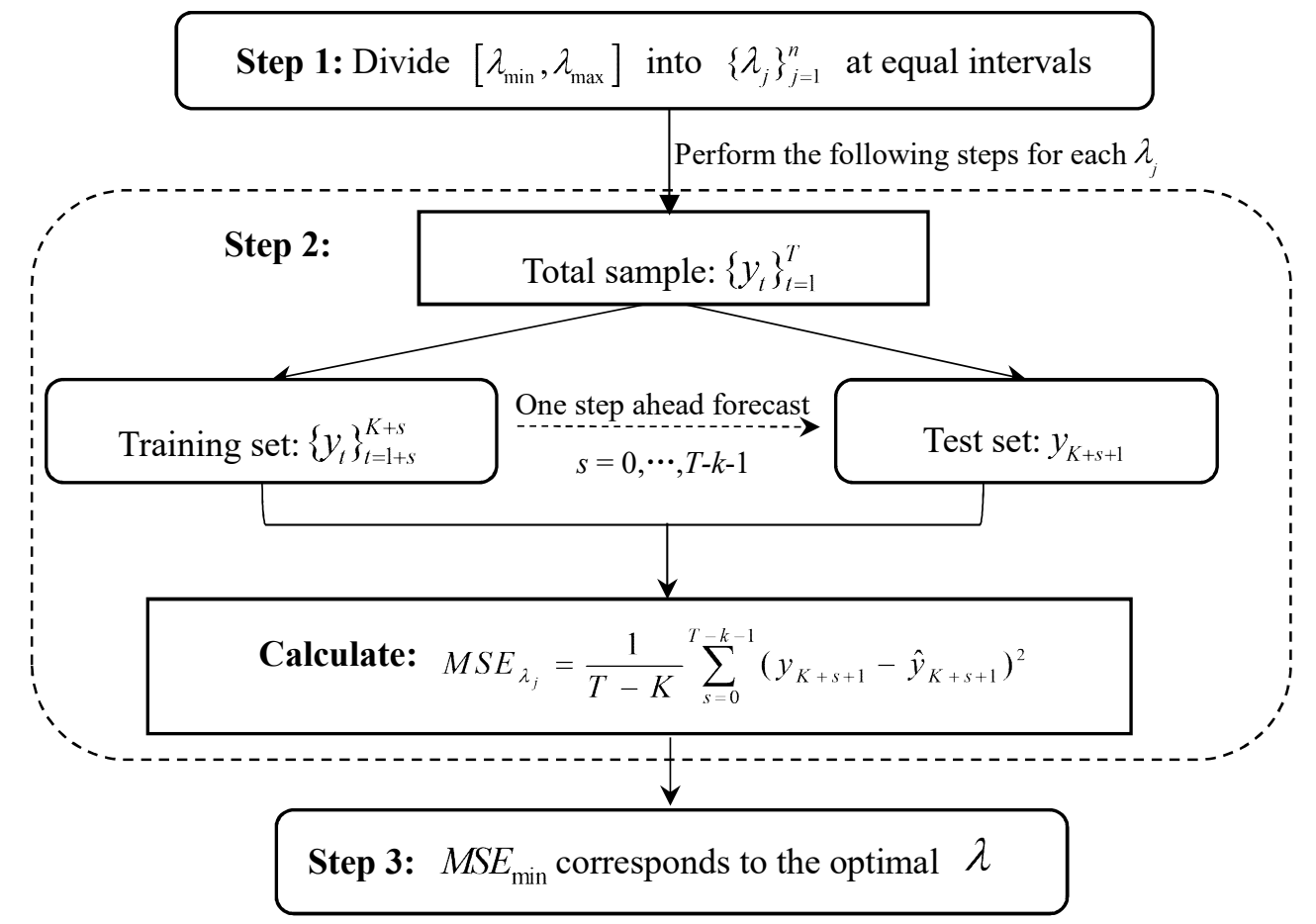

Figure 1. Steps of time series cross-validation.

\subsection{Model prediction evaluation method}

This study first uses root mean square error (hereinafter, RMSE), mean absolute percentage error (hereinafter, MAPE), and mean absolute scaled error (hereinafter, MASE) as evaluation indicators to evaluate the nowcasting power of the LASSO-MIDAS model. RMSE, MAPE, and MASE can be calculated as follows: 


$$
\begin{gathered}
R M S E=\sqrt{\frac{1}{T} \sum_{t=1}^{T}\left(\hat{y}_{t}-y_{t}\right)^{2}}, \\
M A P E=\frac{1}{T} \sum_{t=1}^{T}\left|\frac{\hat{y}_{t}-y_{t} \mid}{y_{t}}\right|, \\
M A S E=\frac{\sum_{t=1}^{T}\left|\hat{y}_{t}-y_{t}\right| / T}{\sum_{t=2}^{T}\left|y_{t}-y_{t-1}\right| /(T-1)},
\end{gathered}
$$

where $T$ is the number of observations, $y_{t}$ denotes the actual data at period $t$, and $\hat{y}_{t}$ is the nowcast value in the same period. Smaller values of RMSE, MAPE, and MASE indicate better nowcasting performance (Li et al., 2018).

RMSE, MAPE, and MASE are used extensively to evaluate the nowcasting performance of models, but it is not suitable for judging which particular model is better solely on the basis of these values when the difference between the nowcast errors of different models is quite small (Diebold and Mariano, 1995). Therefore, this study uses the model confidence set (hereinafter, MCS) test to select the best models, which can examine the prediction statistical errors of various models to avoid model misjudgments caused by a single sample and loss functions. The MCS test can generate a model confidence set that contains the best model(s) with specified confidence, and all the models in the set are superior to those out of the set (Hansen et al., 2011). The procedure of the MCS test is as follows (Ma et al., 2019):

(1) Set $M=M_{0}$;

Consider an initial model set $M_{0}=\left\{1, \ldots, m_{0}\right\}$, where $m_{0}$ represents the number of models. Each model can obtain nowcast accuracy $y_{t}$ in period-t. We denote the loss associated with the model $i$ in period- $t$ as $L_{i, t}$ (Hansen et al., 2011, Ma et al., 2019). We define the relative loss function values as follows:

$$
d_{i j, t} \equiv L_{i, t}-L_{j, t}, \text { for all } i, j \in M_{0} \text {. }
$$

(2) Construct the statistics to test $H_{0, M}$ with the equivalence test $\delta_{m}$ at a specific significance level $\alpha$;

The MCS test consists of an equivalence test $\delta_{m}$ and an elimination rule $e_{m}$ (Hansen et al., 2011, Ma et al., 2019). The null hypotheses of the equivalence test mean that the two models have equal forecast accuracy:

$$
H_{0, M}: E\left(d_{i, j, m}\right)=0, \text { for } \forall i, j \in M \subset M_{0}
$$

We select the range statistic $T_{R}$ for our models following the suggestion of Hansen et al. (2011), which can be expressed as

$$
T_{R}=\max _{i, j \in M} \frac{\left|\bar{d}_{i j}\right|}{\sqrt{\operatorname{var}\left(d_{i j}\right)}},
$$

where $\bar{d}_{i j}$ denotes the mean value of the relative loss function of model $i$ and model $j$. Its variance can be estimated easily through bootstrap methods (Ma et al., 2019).

(3) If $H_{0, M}$ is accepted, then define $\hat{M}_{1-\alpha}^{*}=M$; if not, the elimination rule $e_{m}$ will eliminate the inferior model from $M$.

The above procedure is repeated from Step (2) until the null is accepted, and then we can obtain the set $\hat{M}_{1-\alpha}^{*}$, which is a set of "surviving models" at the significance level $\alpha$ (the model confidence set). For a model $f\left(f \in M_{0}\right)$, if the $p$-value of MCS 
is larger than $\alpha$, then $f \in \hat{M}_{1-\alpha}^{*}$. The closer the $p$-value is to 1 , the more prominent the model's tourism demand prediction performance. Referring to the existing literature, the significance level of the MCS test is set to $\alpha=0.1$ (Hansen et al., 2011). All of the above steps are implemented in R.

\section{Design of the empirical analysis}

In this section, we first introduce the data used in empirical research and then describe the details of the nowcasting strategy.

\subsection{The data}

In order to achieve the purposes of this study, the explanatory variables in the study include two different types of data sources: (1) search engine data - the daily updated Baidu Index; (2) monthly updated official statistical data, including tourism-related and macroeconomic variables. To make the empirical results more credible, this study uses the same Baidu Index data set as that used by Wen et al. (2021) to verify the above purposes. There are two reasons for using the same Baidu Index data set in empirical research. First, Wen et al. (2021) is one of the few studies using search engine data nowcast tourism demand, and the results from the same data set can provide a reliable benchmark. Second, access to this data set is open, so the same data set can be used to test the effectiveness of a proposed method in tourism demand nowcasting or forecasting (Li and Law, 2019). Specifically, using the six aspects of tourism planning (lodging, dining, attractions, transportation, tours, and shopping), 101 keywords in the Baidu Index were collected. The data set covers the period from January 1, 2011, to December 31, 2019, at a daily frequency. The explanatory variables are standardized to control differences in scale. Due to space limitations, we do not report the specific content and the collection methods used for keywords; details can be found in Wen et al. (2021).

Regarding the selection of official statistical data, we choose monthly VAs from mainland China to Hong Kong to measure tourism demand, and the logarithmic transformation was applied. The microeconomic theory suggests that the destination's own price, the price of competitive destinations, and tourist income are the most important factors that affect tourism demand (Song et al., 2003, Song et al., 2009). Some studies indicate that including a wider set of macroeconomic variables may help us to better understand the decision-making of tourists in the source country and the dynamics driving the destination's tourism demand (Song et al., 2011, Chatziantoniou et al., 2016, Lourenço et al., 2020). Therefore, referring to a series of references, this study selects the following tourism-related and macroeconomic data as official statistical data:

(1) The destination's own price variable $P_{н К}=\left(C P I_{н К} / E X_{н К}\right) /\left(C P I_{M L} / E X_{м L}\right)$, which measures the tourism price in Hong Kong relative to that of the respective source market in mainland China, where $C P I_{H K}$ and $C P I_{M L}$ represent the $C P I$ for the destination Hong Kong and the source country mainland China, respectively. $E X_{H K}$ and $E X_{M L}$ are the exchange rates of the destination Hong Kong and the source country mainland China against the US dollar, respectively.

(2) The substitute price variable $P_{S}$, which represents a weighted average index of selected countries' tourism prices. Thailand is the most popular destination for 
Chinese outbound tourism, and Macau has the greatest proximity to Hong Kong as far as both culture and geographical distance are concerned. Therefore, this study chooses Thailand and Macau to calculate the substitute price variable $P_{S}=\sum_{j=1}^{2}\left(C P I_{j, t} / E X R_{j, t}\right) /\left(C P I_{H K, t} / E X R_{H K, t}\right) w_{j, t} \quad, \quad j \in\{$ Macau, Thailand $\} \quad, \quad$ where $w_{j, t}=Q_{j, t} /\left(\sum_{j=1}^{2} Q_{j, t}\right)$ is calculated as the number of VAs from the source market mainland China to the competitive destination $j$ (Thailand or Macau) divided by the number of VAs from mainland China to both competitors of Hong Kong.

(3) The industrial production index $I P_{M L}$, which measures the prosperity and development trend of the industrial economy of mainland China as a proxy of GDP - a more frequently used measure of income - due to the unavailability of monthly frequency data (Chatziantoniou et al., 2016).

(4) $C P I_{M L}$, which measures the general price level or inflation in the source country. Higher inflation will cause travelers to consider reducing unnecessary travel expenditure.

(5) Consumer confidence index $C C_{M L}$, which reflects people's expectations of future economic conditions.

(6) The logarithmic form of the economic policy uncertainty $E P U_{M L}$ proposed by Baker et al. (2016) measures the policy-related uncertainty of the origin country at the fiscal and monetary policy levels.

(7) Consumer price differences indicator $C P D s=C P I_{H K} / C P I_{M L}$, which is used to assess the consumer price differences between the destination Hong Kong and the origin region of mainland China.

The above five indicators $I P_{M L}, C P I_{M L}, C C_{M L}, E P U_{M L}$, and $C P D s$ are used to capture expectations of future economic conditions, which have general influences on the demand for consumer products, including tourism.

(8) The logarithmic form of the lag value of Hong Kong VAs ( $\left.V A_{\text {lags }}\right)$ is included as the expectation and habit variable that affects tourism demand.

The above official statistical data all cover the period from January 2011 to March 2020 at a monthly frequency. To further verify the causal relationships between the above selected explanatory variables (including the search engine data aggregated to the monthly frequency and official statistical data) and the dependent variable VAs, the Dickey-Fuller unit root test, Johansen cointegration test and finally the Granger causality test are implemented. They consistently show supportive evidence on causality. Due to the space constraint, the testing results are omitted here but available upon request from the authors.

\subsection{Nowcasting strategy}

One of the purposes of this study is to examine whether compared with the factor-type MIDAS model, the LASSO-MIDAS model is more applicable and effective for nowcasting tourism demand.

In this study, two types of factor models are selected as comparative models. The first one is the factor-augmented MIDAS (hereinafter, Fa-MIDAS) model proposed by Ferrara and Marsilli (2019). The second one is the GDFM model proposed by Forni et al. (2005) to nowcast VAs in Hong Kong. In this paper, the GDFM-MIDAS model is constructed by combining the GDFM with the MIDAS model. In addition, the 
low-frequency factor ordinary least squares (hereinafter, Fa-OLS), GDFM-OLS, and LASSO-OLS models are selected as the corresponding benchmark models for Fa-MIDAS, GDFM-MIDAS, and LASSO-MIDAS models, respectively, to test whether the MIDAS-type model is superior in nowcasting over the same frequency model. Specifically, the Fa-type and GDFM-type models used in this study require a two-step process: the first step is to extract the factors from all explanatory variables, and the second step is to use the extracted factors as the explanatory variables in the OLS or MIDAS model to nowcast the dependent variable (Marsilli, 2014). It should be noted that, first, when using low-frequency OLS models for nowcasting, we use the univariate autoregressive model to fill in the missing data within the nowcast month, and then use the Fa-OLS, GDFM-OLS, and LASSO-OLS models to generate nowcasts based on low-frequency explanatory variables aggregated from high-frequency variables (Soybilgen and Yazgan, 2018). Second, the number of factors in the Fa-OLS and Fa-MIDAS models is determined on the basis of an information criterion proposed by Bai and $\mathrm{Ng}$ (2002). Third, the GDFM-OLS and GDFM-MIDAS models use a formal test proposed by Hallin and Liška (2007) to determine the number of factors. Fourth, the optimal lag order of the explanatory variables in all models is determined by the AIC and BIC criteria.

On the basis of the above comparison model, this study analyzes the applicability and effectiveness of the LASSO-MIDAS model for nowcasting Hong Kong tourism demand. Specifically, the first step is to divide the total sample into two parts: (1) the training set: data covering the period from 2011M1 to $2018 \mathrm{M} 12$ are used for in-sample estimation; (2) the test set: data covering the period from 2019M1 to 2019M12 are used to evaluate the nowcasting performance. Then, an expanding window method is used to generate one-step-ahead nowcasts recursively. Essentially, the data period used for estimation is expanded by one more month each time to generate a new nowcast over the test set. Different nowcasts are generated using data up to different numbers of days for that month. For example, data available until the first day of that specific month is used to generate the first nowcast, and this is repeated to generate 12 nowcasts and its nowcasting performance is evaluated using the test set.

\subsection{Inconsistent data release time}

Hong Kong VAs data are usually released with a month lag. As shown in Figure 2, the VAs data for January 2019 were first published on March 1, 2019. As the daily Baidu Index data are updated in real time, when the Baidu Index data on January 1 are available, we can add them to the model to nowcast VAs for January. This process is repeated until the end of the month to generate a total of 31 nowcasting results for the month. 


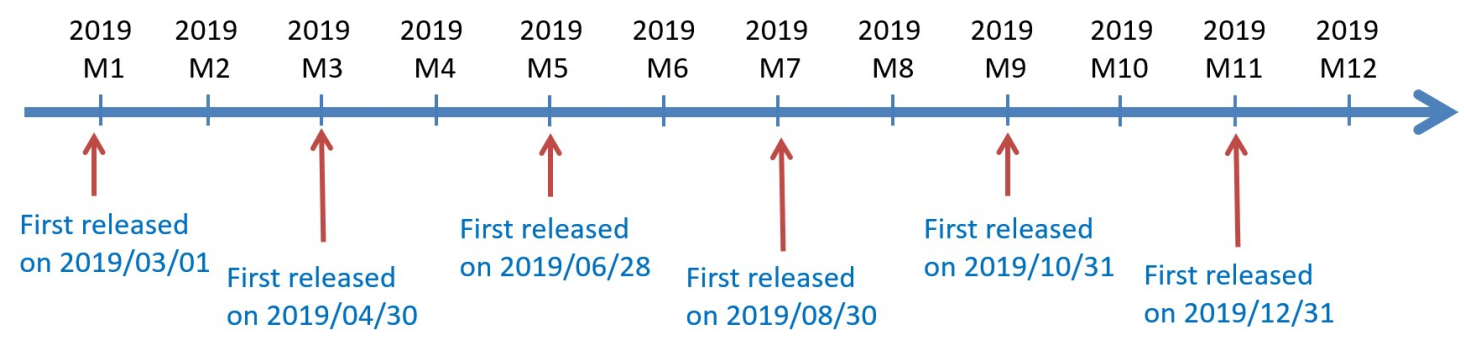

Figure 2. The first release date of monthly VAs data from 2019M1 to 2019M12.

Compared with the daily updated Baidu Index data, the release of monthly official statistical data has different lags for different variables. Tourism demand nowcasting needs to know the specific release dates of the official statistical data in order to incorporate the latest available information. Therefore, we summarize the release schedule of official statistical data in the latest year in Table 1. It should be noted that the EPU data website (http://www.policyuncertainty.com/) does not provide a specific release date for the data. We only observed that the EPU value in May was updated on June 4, so we unified the release date of the EPU data to the fourth day of each month. According to Table 1, the timeline for adding official statistical data to the nowcast process can be summarized as $E P U_{M L} \rightarrow C P I_{M L} \rightarrow I P_{M L} \rightarrow C P D s, P_{H K} \rightarrow$ $P_{S} \rightarrow C C I_{M L} \rightarrow V A_{\text {lags }}$; the adding order of $V A_{\text {lags }}$ and $C C I_{M L}$ can be adjusted according to Table 1 . 
Table 1. First release dates of monthly official statistical data from 2019M1 to 2019M12.

\begin{tabular}{l|llllllll}
\hline Month & $E P U_{M L}$ & $C P I_{M L}$ & $I P_{M L}$ & $C P D s$ & $P_{H K}$ & $P_{s}$ & $C C I_{M L}$ & $V_{\text {lags }}$ \\
\hline 2019M1 & $2019 / 02 / 04$ & $2019 / 02 / 10$ & $2019 / 03 / 17$ & $2019 / 02 / 20$ & $2019 / 02 / 20$ & $2019 / 02 / 28$ & $2019 / 03 / 30$ & $2019 / 02 / 28$ \\
2019M2 & $2019 / 03 / 04$ & $2019 / 03 / 10$ & $2019 / 03 / 16$ & $2019 / 03 / 23$ & $2019 / 03 / 23$ & $2019 / 03 / 23$ & $2019 / 03 / 30$ & $2019 / 03 / 31$ \\
2019M3 & $2019 / 04 / 04$ & $2019 / 04 / 10$ & $2019 / 04 / 17$ & $2019 / 04 / 23$ & $2019 / 04 / 23$ & $2019 / 04 / 28$ & $2019 / 04 / 30$ & $2019 / 04 / 29$ \\
2019M4 & $2019 / 05 / 04$ & $2019 / 05 / 09$ & $2019 / 05 / 15$ & $2019 / 05 / 21$ & $2019 / 05 / 21$ & $2019 / 05 / 24$ & $2019 / 05 / 29$ & $2019 / 05 / 31$ \\
2019M5 & $2019 / 06 / 04$ & $2019 / 06 / 12$ & $2019 / 06 / 14$ & $2019 / 06 / 21$ & $2019 / 06 / 21$ & $2019 / 06 / 21$ & $2019 / 07 / 02$ & $2019 / 06 / 28$ \\
2019M6 & $2019 / 07 / 04$ & $2019 / 07 / 10$ & $2019 / 07 / 15$ & $2019 / 07 / 22$ & $2019 / 07 / 22$ & $2019 / 07 / 22$ & $2019 / 07 / 31$ & $2019 / 08 / 01$ \\
2019M7 & $2019 / 08 / 04$ & $2019 / 08 / 09$ & $2019 / 08 / 15$ & $2019 / 08 / 20$ & $2019 / 08 / 20$ & $2019 / 08 / 26$ & $2019 / 09 / 02$ & $2019 / 08 / 30$ \\
2019M8 & $2019 / 09 / 04$ & $2019 / 09 / 10$ & $2019 / 09 / 16$ & $2019 / 09 / 20$ & $2019 / 09 / 20$ & $2019 / 09 / 25$ & $2019 / 09 / 29$ & $2019 / 09 / 30$ \\
2019M9 & $2019 / 10 / 04$ & $2019 / 10 / 15$ & $2019 / 10 / 18$ & $2019 / 10 / 22$ & $2019 / 10 / 22$ & $2019 / 10 / 22$ & $2019 / 10 / 31$ & $2019 / 10 / 31$ \\
2019M10 & $2019 / 11 / 04$ & $2019 / 11 / 09$ & $2019 / 11 / 14$ & $2019 / 11 / 21$ & $2019 / 11 / 21$ & $2019 / 11 / 25$ & $2019 / 12 / 03$ & $2019 / 11 / 29$ \\
2019M11 & $2019 / 12 / 04$ & $2019 / 12 / 10$ & $2019 / 12 / 16$ & $2019 / 12 / 20$ & $2019 / 12 / 20$ & $2019 / 12 / 26$ & $2019 / 12 / 31$ & $2019 / 12 / 31$ \\
2019M12 & $2020 / 01 / 04$ & $2020 / 01 / 09$ & $2020 / 01 / 17$ & $2020 / 01 / 21$ & $2020 / 01 / 21$ & $2020 / 01 / 23$ & $2020 / 02 / 07$ & $2020 / 01 / 31$ \\
\hline
\end{tabular}

Note: The official statistical data release dates are obtained from the CEInet Statistics Database, the Wind Database, and the CEIC database.

The different release lags of official statistical data lead to a non-balanced data set for each time point and thus a ragged-edge data set. Due to the different times at which the explanatory variables can be available for nowcasting, we consider different models for each day of the month and finally get 31 different models. Each model includes the currently available variables. The specific details are provided in Table 2. In the actual nowcast process, the variables available for each model are brought into FA-OLS, GDFM-OLS, LASSO-OLS, Fa-MIDAS, GDFM-MIDAS, and LASSO-MIDAS models to nowcast Hong Kong VAs.

In addition, this study also uses only monthly official statistical data to nowcast tourism demand without including the daily Baidu Index data to examine whether and when the Baidu Index data can provide additional information that is not included in the official data. On the basis of the release date of the official statistical data, we list eight models that contain only currently available official statistical data in Table 3 . In addition, it should be noted that the official statistical data are consistent with the explained variable, VAs, and are released on a monthly basis. The same frequency models including Fa-OLS, GDFM-OLS, and LASSO-OLS models can be directly used for nowcasting monthly frequency data, without using MIDAS-type models. 
Table 2. Equations of the 31 models $(M 1, \ldots$, M31) used to nowcast VAs over each month.

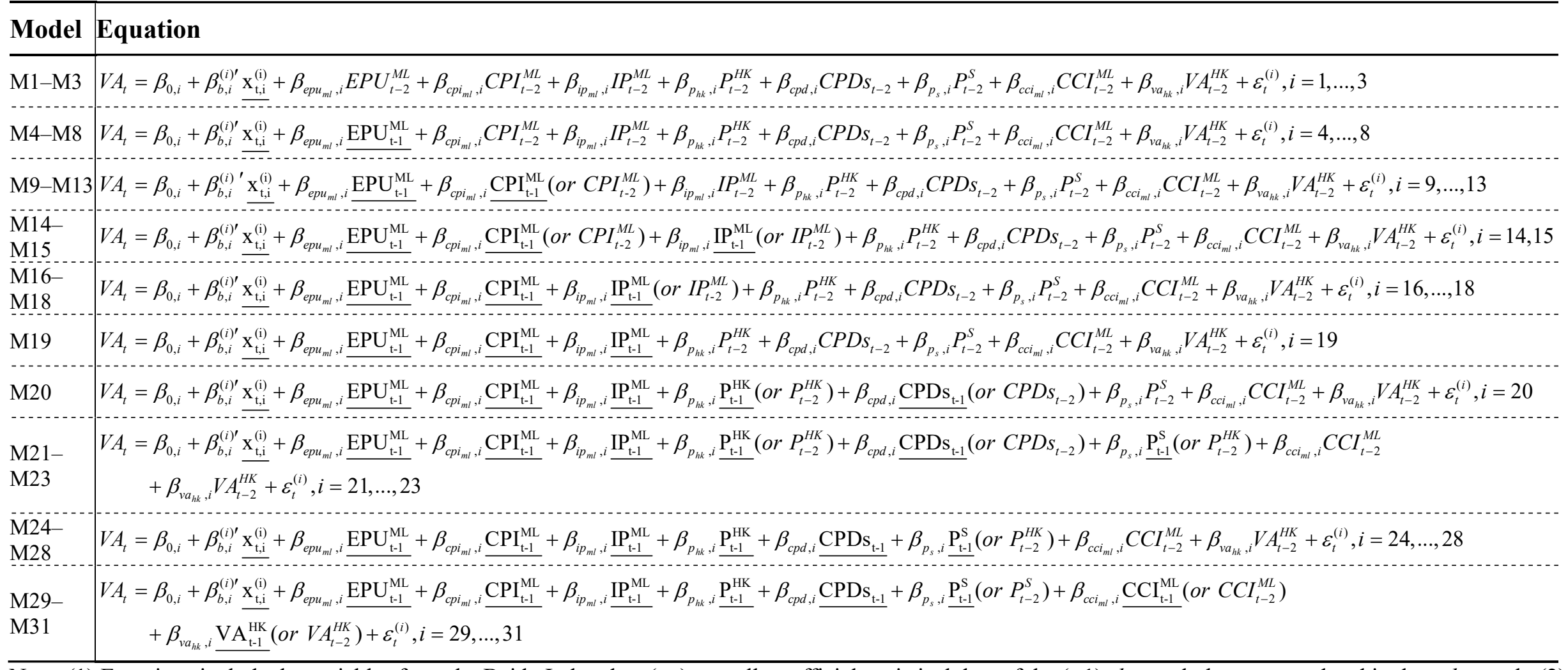

Note: (1) Equations include the variables from the Baidu Index data $\left(x_{t}\right)$ as well as official statistical data of the (t-1)-th month that were updated in the $t$-th month. (2) Since the release date of the official statistical data in each month is different, the specific number of periods that variables can be added to the formula is determined according to the specific release date of the variable in Table 1. (3) The underlined part of the formula indicates the latest updated data that can be added in the current period. 
Table 3. Equations of the eight models (Official1,..., Official 8 ) used to nowcast VAs over each month.

\begin{tabular}{|c|c|}
\hline Model & Equation \\
\hline Official $_{1}$ & $V A_{t}=\beta_{0,1}+\beta_{c p u_{m l}, 1} E P U_{t-2}^{M L}+\beta_{c p i_{m l}, 1} C P I_{t-2}^{M L}+\beta_{i p_{m l}, 1} I P_{t-2}^{M L}+\beta_{p_{h k}, 1} P_{t-2}^{H K}+\beta_{c p d_{s}, 1} C P D s_{t-2}+\beta_{p_{s}, 1} P_{t-2}^{S}+\beta_{c c i, 1} C C I_{t-2}^{M L}+\beta_{v a_{h k}, 1} V A_{t-2}^{H K}+\varepsilon_{t}$ \\
\hline Official $_{2}$ & $V A_{t}=\beta_{0,1}+\beta_{e p u_{m l},} \underline{\mathrm{EPU}_{\mathrm{t}-1}^{\mathrm{ML}}}+\beta_{c i_{m_{l},},} C P I_{t-2}^{M L}+\beta_{i p_{m l}, 1} I P_{t-2}^{M L}+\beta_{p_{h k}, 1} P_{t-2}^{H K}+\beta_{c p d_{s}, 1} C P D s_{t-2}+\beta_{p_{s}, 1} P_{t-2}^{S}+\beta_{c c i, 1} C C I_{t-2}^{M L}+\beta_{v a_{h k}, 1} V A_{t-2}^{H K}+\varepsilon_{t}$ \\
\hline Official $3_{3}$ & $V A_{t}=\beta_{0,2}+\beta_{c p u_{m l}, 2} \underline{\mathrm{EPU}_{\mathrm{t}-1}^{\mathrm{ML}}}+\beta_{c p_{m l}, 2} \underline{\mathrm{CPI}_{\mathrm{t}-1}^{\mathrm{ML}}}+\beta_{i p_{m l}, 2} I P_{t-2}^{M L}+\beta_{p_{h k}, 2} P_{t-2}^{H K}+\beta_{c p d_{s}, 2} C P D s_{t-2}+\beta_{p_{s}, 2} P_{t-2}^{S}+\beta_{c c i, 2} C C I_{t-2}^{M L}+\beta_{v a_{h k}, 2} V A_{t-2}^{H K}+\varepsilon_{t}$ \\
\hline Official $4_{4}$ & $V A_{t}=\beta_{0,3}+\beta_{e p u_{m l}, 3} \underline{\mathrm{EPU}_{\mathrm{t}-1}^{\mathrm{ML}}}+\beta_{c p i_{m l}, 3} \underline{\mathrm{CPI}_{\mathrm{t}-1}^{\mathrm{ML}}}+\beta_{i p_{m l}, 3} \underline{\mathrm{IP}_{\mathrm{t}-1}^{\mathrm{ML}}}+\beta_{p_{l k}, 3} P_{t-2}^{H K}+\beta_{c p d_{s}, 3} C P D s_{t-2}+\beta_{p_{s}, 3} P_{t-2}^{S}+\beta_{c c i, 3} C C I_{t-2}^{M L}+\beta_{v a_{h k}, 3} V A_{t-2}^{H K}+\varepsilon_{t}$ \\
\hline Official $_{5}$ & $V A_{t}=\beta_{0,4}+\beta_{e p u_{m l}, 4} \underline{\mathrm{EPU}_{\mathrm{t}-1}^{\mathrm{ML}}}+\beta_{c p i_{m l}, 4} \underline{\mathrm{CPI}_{\mathrm{t}-1}^{\mathrm{ML}}}+\beta_{i p_{m l}, 4} \underline{\mathrm{IP}_{\mathrm{t}-1}^{\mathrm{ML}}}+\beta_{p_{h k}, 4} \underline{\mathrm{P}_{\mathrm{t}-1}^{\mathrm{HK}}}+\beta_{c p d_{s}, 4} \underline{\mathrm{CPDs}_{\mathrm{t}-1}}+\beta_{p_{s}, 4} P_{t-2}^{S}+\beta_{c c i, 4} C C I_{t-2}^{M L}+\beta_{v a_{h k}, 4} V A_{t-2}^{H K}+\varepsilon_{t}$ \\
\hline Official $6_{6}$ & $V A_{t}=\beta_{0,5}+\beta_{e p u_{m l}, 5} \underline{\mathrm{EPU}_{\mathrm{t}-1}^{\mathrm{ML}}}+\beta_{c p_{m l}, 5} \underline{\mathrm{CPI}_{\mathrm{t}-1}^{\mathrm{ML}}}+\beta_{i p_{m l}, 5} \underline{\mathrm{IP}_{\mathrm{t}-1}^{\mathrm{ML}}}+\beta_{p_{h k}, 5} \underline{\mathrm{P}_{\mathrm{t}-1}^{\mathrm{HK}}}+\beta_{c p d_{s}, 5} \underline{\mathrm{CPDs}_{\mathrm{t}-1}}+\beta_{p_{s}, 5} \underline{\mathrm{P}_{\mathrm{t}-1}^{\mathrm{S}}}+\beta_{c c i, 4} C C I_{t-2}^{M L}+\beta_{v a_{h k}, 4} V A_{t-2}^{H K}+\varepsilon_{t}$ \\
\hline Official $_{7}$ & 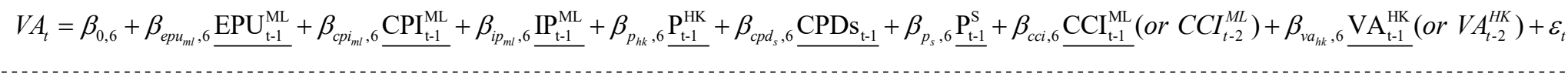 \\
\hline Official $_{8}$ & $V A_{t}=\beta_{0,6}+\beta_{e p u_{m l}, 6} \underline{\mathrm{EPU}_{\mathrm{t}-1}^{\mathrm{ML}}}+\beta_{c p i_{m l}, 6} \underline{\mathrm{CPI}_{\mathrm{t}-1}^{\mathrm{ML}}}+\beta_{i p_{m l},} \underline{\mathrm{IP}_{\mathrm{t}-1}^{\mathrm{ML}}}+\beta_{p_{h k}, 6} \underline{\mathrm{P}_{\mathrm{t}-1}^{\mathrm{HK}}}+\beta_{c p d_{s}, 6} \underline{\mathrm{CPDs}_{\mathrm{t}-1}}+\beta_{p_{s}, 6} \underline{\mathrm{P}_{\mathrm{t}-1}^{\mathrm{S}}}+\beta_{c c i, 6} \underline{\mathrm{CCI}_{\mathrm{t}-1}^{\mathrm{ML}}}\left(\right.$ or $\left.C C I_{t-2}^{M L}\right)+\beta_{v a_{h k}, 6} \mathrm{VA}_{\mathrm{t}-1}^{\mathrm{HK}}\left(o r V A_{t-2}^{H K}\right)+\varepsilon_{t}$ \\
\hline
\end{tabular}

Note: The underlined part of the formula indicates the latest updated data that can be added in the current period. 


\section{Empirical results}

In this section, we show the empirical research results of using the target model LASSO-MIDAS and its comparative models to nowcast VAs from mainland China to Hong Kong using different data sources. First, this study shows the nowcasting results using three various data sources in a pseudo-real-time framework. The purpose is to investigate whether the real-time updated Baidu Index data can be useful for improving the nowcasting accuracy of VAs, and when, once we have obtained the official statistical data. Second, this study uses the MCS method to test the model's prediction difference in a statistical sense. Finally, this study shows a series of variable selection results that are most relevant to the explained variable selected by the LASSO-MIDAS model during the nowcasting process. It should be noted that the empirical results based on the MASE and MAPE evaluation indicators are similar to the results based on the RMSE evaluation indicator. Due to space constraints, we mainly describe the results based on the RMSE evaluation indicator. The related results of MAPE and MASE evaluation indicators can be seen in Figures A1-A4 of Appendix A.

\subsection{Is there a gain from using Baidu data and if so when?}

Figures 3(a)-(f) show the RMSE results of six models using three different data sources to nowcast Hong Kong VAs from 2019M1 to 2019M12. The three data sources are (1) daily updated Baidu Index data only, (2) daily updated Baidu Index data + monthly updated official statistical data, and (3) monthly updated official statistical data only. Our purpose is to examine whether real-time updated information such as the Baidu Index data is useful for improving VAs nowcasting accuracy, and if so when. Also, once official statistical data on tourism demand are available, will the accuracy of VAs nowcasting change? It should be noted from Table 1 that official statistical data are released at different times each month. For a comparative analysis, the official statistical data results added in this study are the same in each data update period.

The key empirical results can be summarized as follows. First, compared with the results obtained from Fa-OLS, GDFM-OLS, and LASSO-OLS models, MIDAS-type models significantly improve the nowcasting accuracy in the case of using Baidu Index data and using Baidu Index data combined with official statistical data, which confirms the superiority of the MIDAS model in analyzing mixed-frequency data. Second, the nowcasting results of the Fa-MIDAS model, the GDFM-MIDAS model, and the LASSO-MIDAS model in Figure 3 show that the nowcasting results only using monthly official statistical data are slightly better than those obtained only using the daily Baidu Index data. In addition, the nowcasting accuracy is further improved when the real-time updated monthly official statistical data are gradually added to the nowcasting model using the daily Baidu Index data. Third, the LASSO-MIDAS model is relatively more reliable than the factor-type MIDAS models. A more accurate nowcasting result is obtained by using the 
combination of Baidu Index data and official statistical data, which shows that the variable selection function of the LASSO-MIDAS model can remove redundant variables and retain important explanatory variables to improve the nowcasting performance.
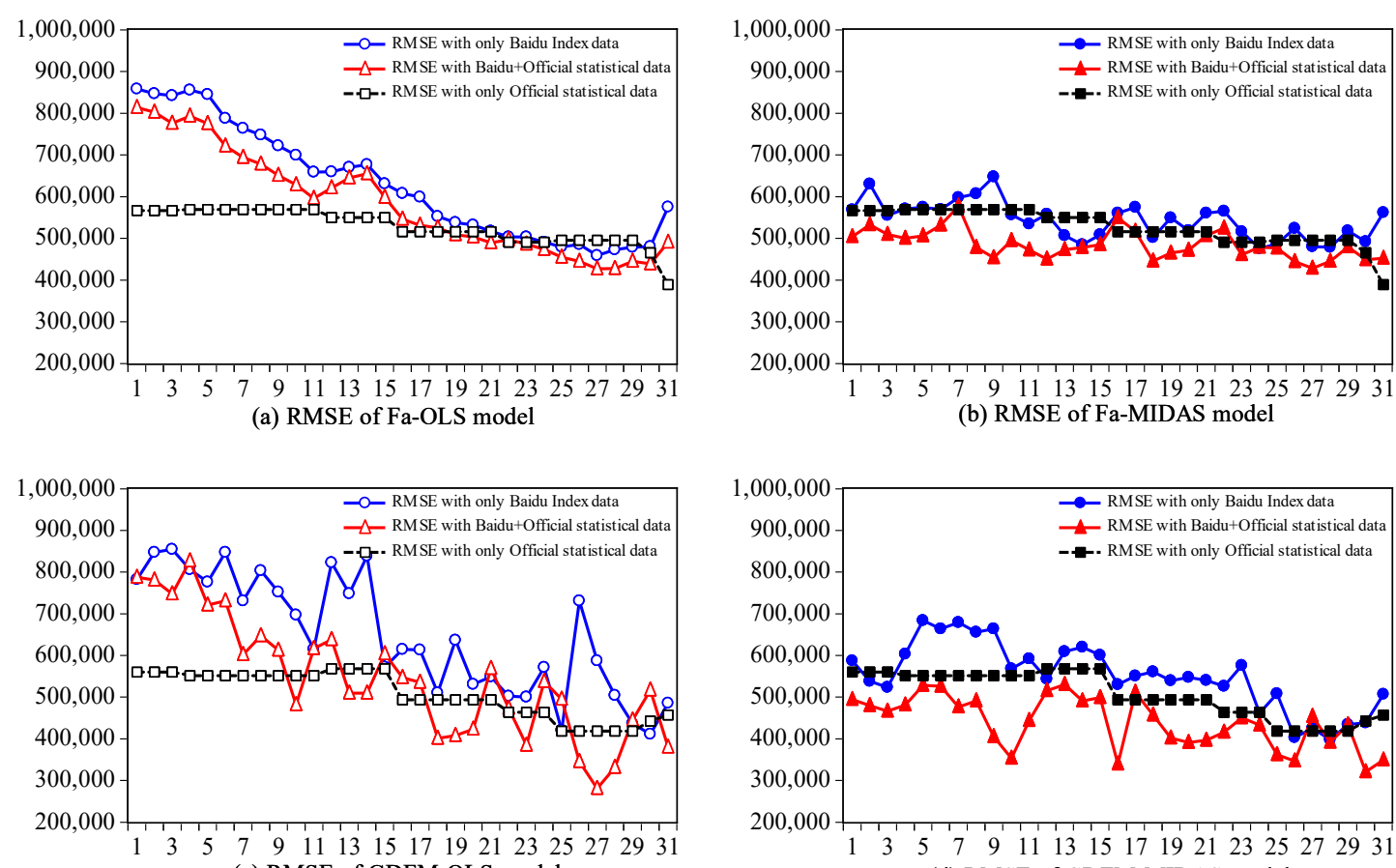

(c) RMSE of GDFM-OLS mode (d) RMSE of GDFM-MIDAS model
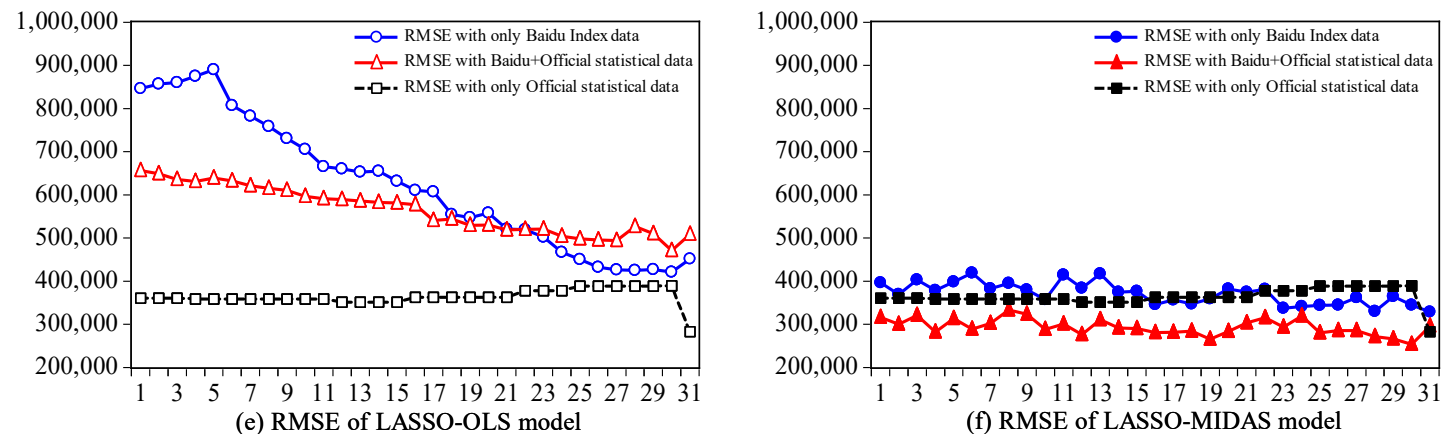

Figure 3. Comparison of nowcasts' RMSE using three different data sources for the same model.

\subsection{Has the accuracy of nowcasting improved as a result of using the}

\section{LASSO-MIDAS model?}

Figures 4 shows the RMSE results of the LASSO-MIDAS model and its comparative model using the same data source to nowcast Hong Kong VAs from 2019M1 to 2019M12. The purpose is to examine the applicability and effectiveness of the LASSO-MIDAS model in tourism demand nowcasting.

The RMSE results of using three different data sources for nowcasting in Figures 
4 (a)-(c) show that, first, the nowcasting accuracy of Fa-MIDAS, GDFM-MIDAS, and LASSO-MIDAS models are better than their corresponding low-frequency models Fa-OLS, GDFM-OLS, and LASSO-OLS. Second, the nowcasting accuracy of the Fa-MIDAS model and the GDFM-MIDAS model are similar, while the nowcasting accuracy of the LASSO-MIDAS model is significantly higher than that of the other competing models. The effectiveness of the LASSO-MIDAS model applied to tourism demand nowcasting is confirmed.

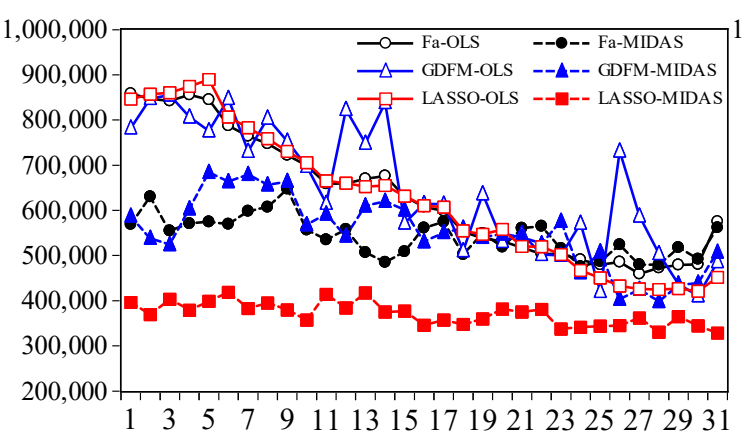

(a) RMSE with only Baidu Index data

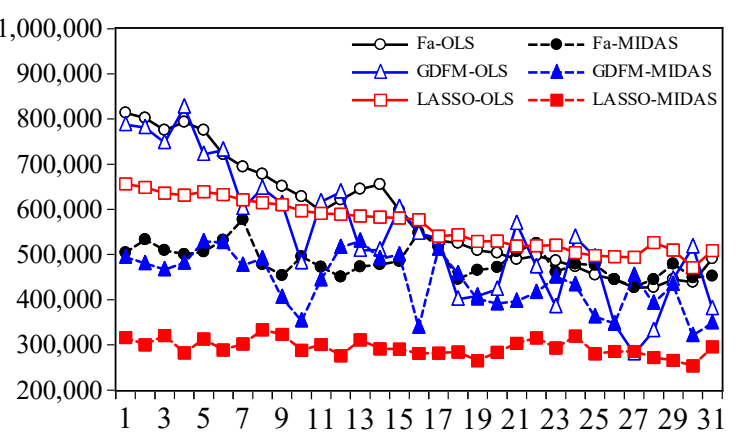

(b) RMSE with Baidu Index + Official statistical data

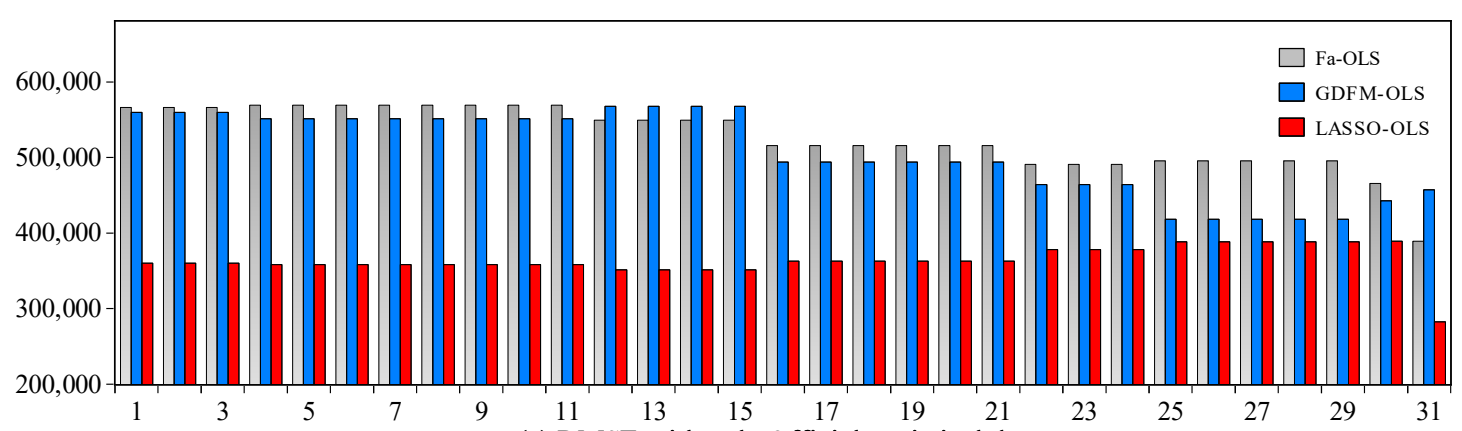

(c) RMSE with only Official statistical data

Figure 4. Comparison of nowcasts' RMSE of different models using the same data source.

The above nowcasting results show that the LASSO-MIDAS model performs best. In order to test whether the differences in nowcast error are statistically significant, this paper also implements the MCS method based on the RMSE, MAPE and MASE measures to test the nowcasting ability of the models. The MCS results in Table 4 suggest that in the two cases of Baidu Index data and the combination of Baidu Index data and official statistical data, the LASSO-MIDAS model is never excluded from the superior model selection. The larger $p$-values of the LASSO-MIDAS model also indicate that the model has much better prediction performance. In addition, the official statistical data are consistent with the explained variable, VAs, and are released on a monthly basis, the same frequency models including Fa-OLS, GDFM-OLS, and LASSO-OLS models can be directly used for nowcasting monthly frequency data, without using MIDAS-type models. Therefore, only the MCS test results of the same frequency model are available when using official statistical data for nowcasting. The MCS results show that the LASSO-OLS 
model performs best when using official statistical data for nowcasting. Overall, the results of the MCS test further statistically confirm the best performance of the LASSO-MIDAS model and its effectiveness in tourism demand nowcasting.

Table 4. Model confidence set test results.

(a) MCS test results - $T_{R}$ statistics based on RMSE

\begin{tabular}{l|llllll}
\hline Model & FA- & GDFM- & LASSO- & Fa- & GDFM- & LASSO- \\
Data & OLS & OLS & OLS & MIDAS & MIDAS & MIDAS \\
\hline Baidu Index data & 0.0000 & 0.0000 & 0.0000 & 0.0000 & 0.0000 & 1.0000 \\
Baidu + Official statistical data & 0.0000 & 0.0000 & 0.0000 & 0.0000 & 0.0000 & 1.0000 \\
Official statistical data & 0.0000 & 0.0000 & 1.0000 & - & - & - \\
\hline \hline
\end{tabular}

(b) MCS test results- $\mathrm{T}_{\mathrm{R}}$ statistics based on MAPE

\begin{tabular}{l|llllll}
\hline $\begin{array}{l}\text { Modet } \\
\text { Data }\end{array}$ & FA- & GDFM- & LASSO- & Fa- & GDFM- & LASSO- \\
Baidu Index data & 0.0000 & 0.0000 & 0.0000 & 0.0000 & 0.0000 & 1.0000 \\
Baidu + Official statistical data & 0.0000 & 0.0000 & 0.0000 & 0.0000 & 0.0000 & 1.0000 \\
Official statistical data & 0.0000 & 0.0000 & 1.0000 & - & - & - \\
\hline \hline
\end{tabular}

(c) MCS test results- $\mathrm{T}_{\mathrm{R}}$ statistics based on MASE

\begin{tabular}{l|llllll}
\hline $\begin{array}{l}\text { Model } \\
\text { Data }\end{array}$ & FA- & GDFM- & LASSO- & Fa- & GDFM- & LASSO- \\
Baidu Index data & 0.0000 & 0.0000 & 0.0000 & 0.0000 & 0.0000 & 1.0000 \\
Baidu + Official statistical data & 0.0000 & 0.0000 & 0.0000 & 0.0000 & 0.0000 & 1.0000 \\
Official statistical data & 0.0000 & 0.0000 & 1.0000 & - & - & - \\
\hline
\end{tabular}

Note: The closer the $p$-value is to 1 , the more prominent the model's tourism demand prediction performance.

\subsection{Variable selection results}

The above results confirm that the LASSO-MIDAS model significantly improves the accuracy of tourism demand nowcasting. One of the advantages of this method is that it can select the variables that play an essential role in VAs nowcasting by constraining the coefficients of variables that have no significant impact on 0 . The change of crucial influencing variables in different periods reflects the evolution of tourism demand to a certain extent, which avoids the difficulty of explaining the significance of estimation factors. We further analyze whether the LASSO-MIDAS model has successfully identified essential variables related to tourism demand from the variable selection results. It should be noted that the nowcast set range selected in this study is from 2019M1 to 2019M12. Twelve variable selection maps (see Fig. 5(a)) can be obtained when nowcasting VAs from January 2019 to December 2019, and a total of 36 variable selection maps can be obtained by using three data sources for nowcasting. Due to space constraints, we only show the results of variable selection in the first period of 2019M1 and the last period of 2019M12 in the two cases of using daily updated Baidu Index data only and using a combination of daily updated Baidu Index data and monthly updated official statistical data. 
Figures 5 and 6 report the variable selection results of the LASSO-MIDAS model in 2019M1 and 2019M12 when using only the daily updated Baidu Index data for nowcasting and when both the daily updated Baidu Index data and upcoming monthly official statistical data are included, respectively. The results show that the selected keywords exist in all six aspects of tourism planning-lodging, dining, attractions, transportation, tours, and shopping. The results indicate that the web search keywords chosen on the basis of the intuition and prior knowledge of the researchers are reasonable; the keywords in the six areas all provide useful information for tourism demand nowcasting. In addition, in the case of using daily Baidu Index data and monthly official statistical data for nowcasting as shown in Figure 6, the gradually updated official statistical data are also recognized by the LASSO-MIDAS model as core variables that affect tourism demand. This shows that the available official statistical data do provide useful information for tourism demand nowcasting. Although the real-time updated Baidu Index data improve the nowcasting accuracy, the official statistical data should not be ignored. The combination of the two types of data leads to more accurate nowcasts. 


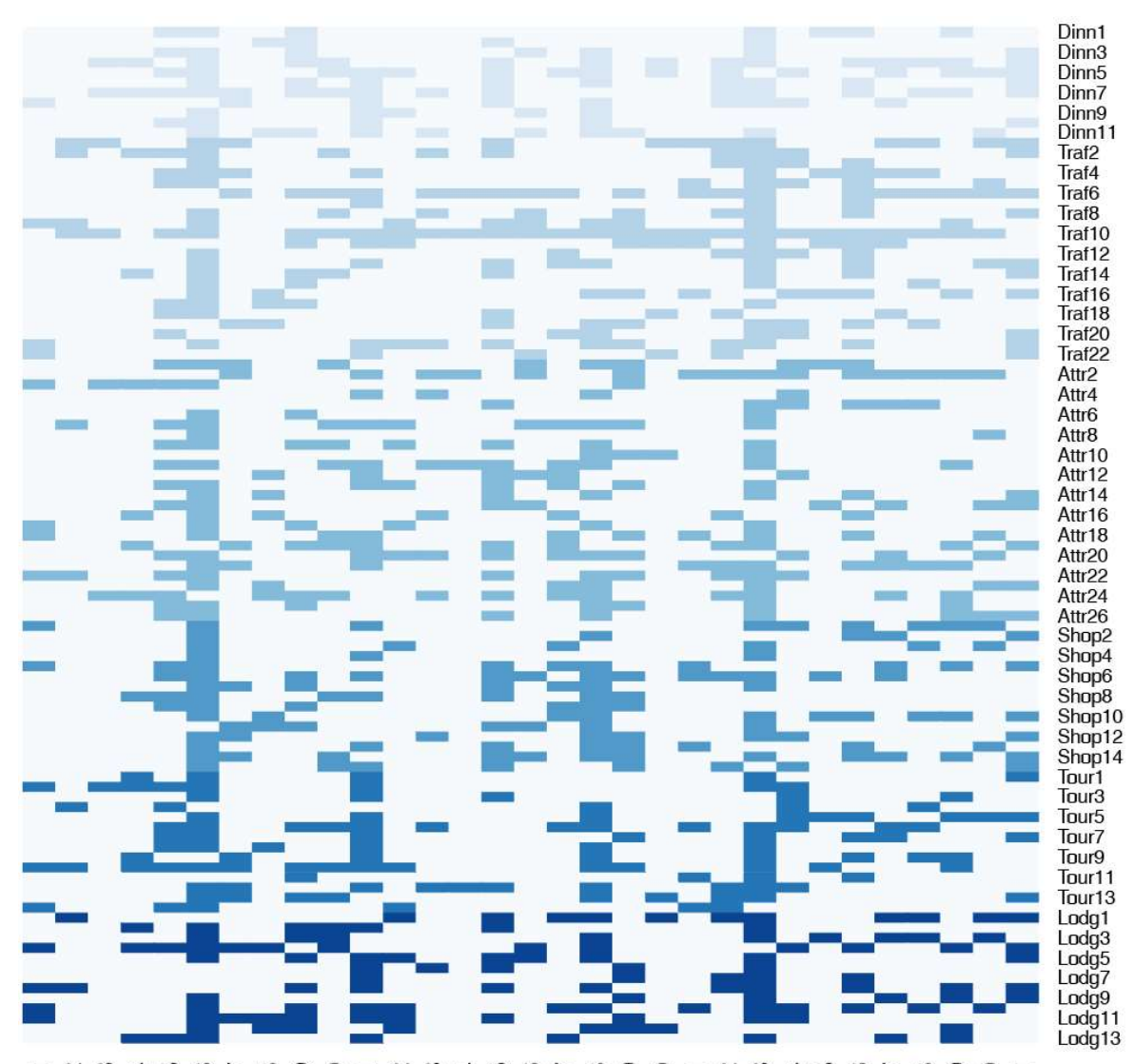

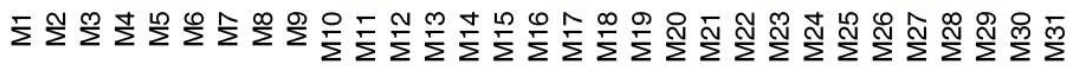

(a) Variable selection results in 2019M1

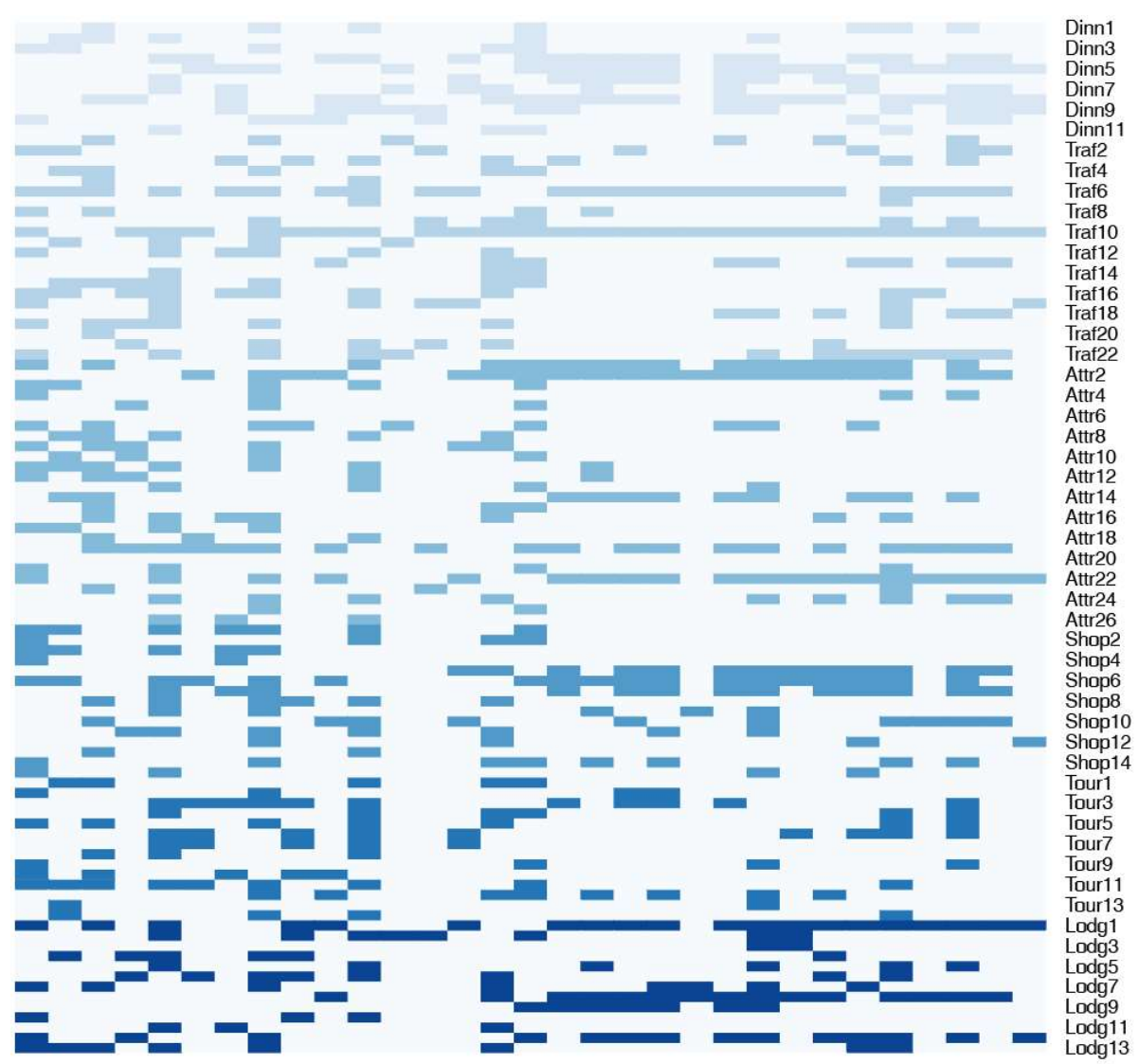

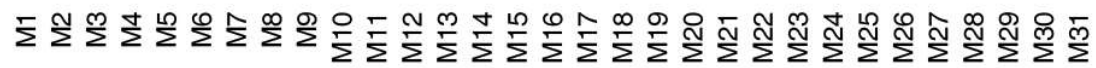

(b) Variable selection results in 2019M12

Figure 5. The variable selection results of the LASSO-MIDAS model when only 101 Baidu Index data are included. 


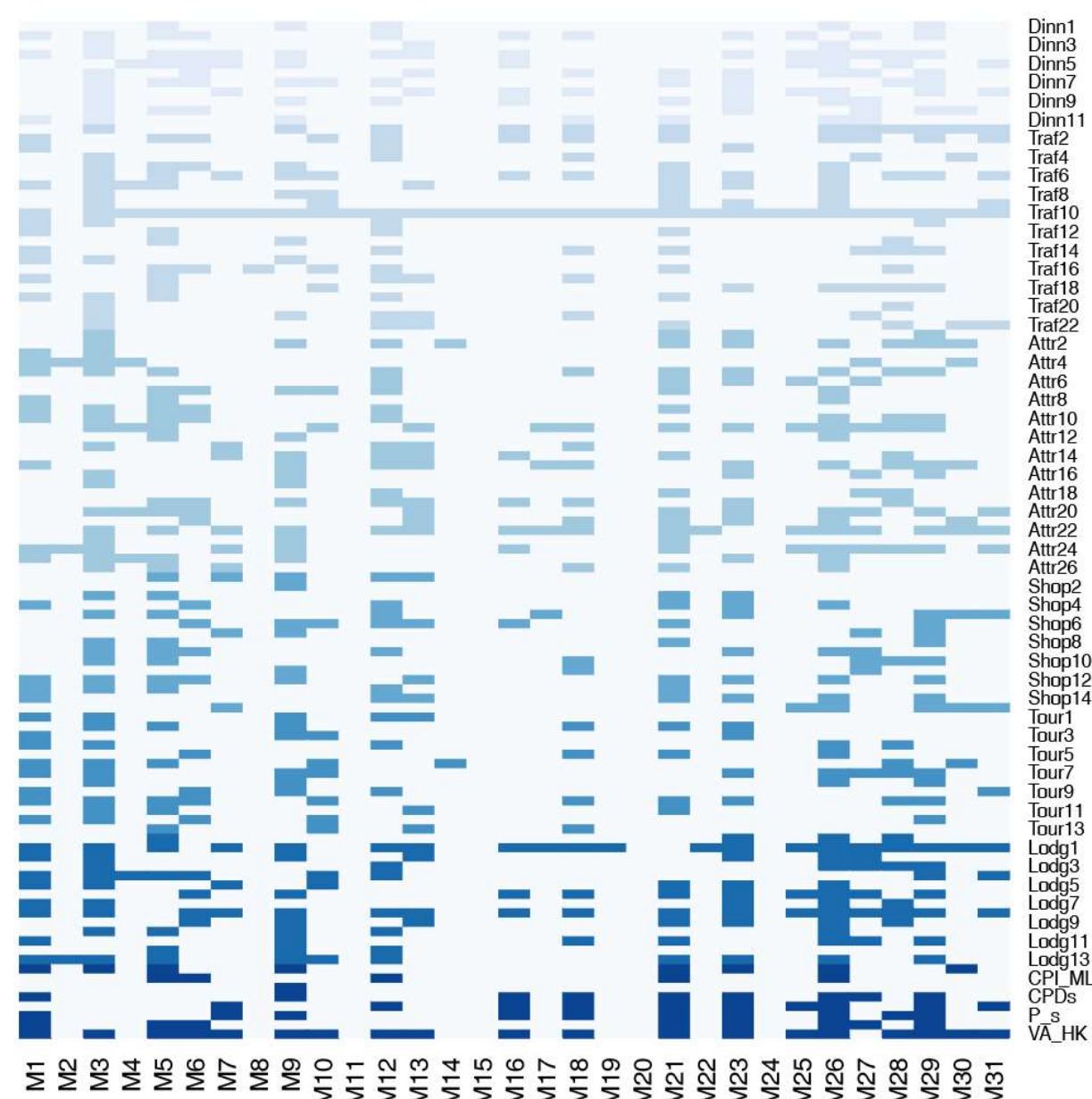

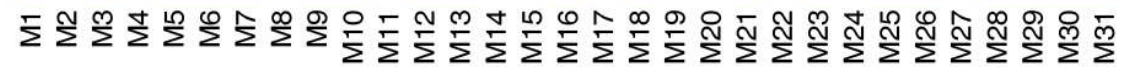

(a) Variable selection results in 2019M1

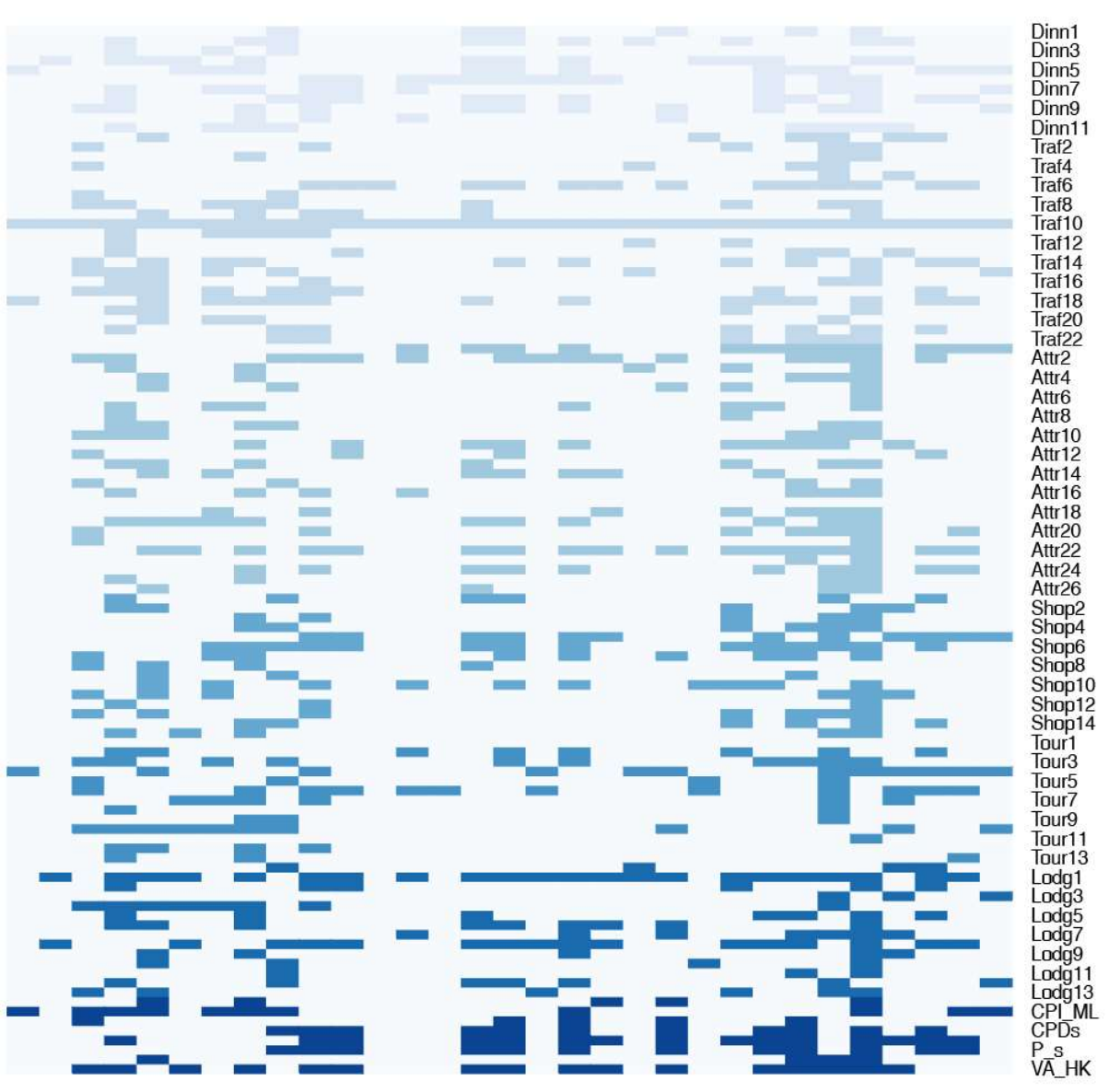

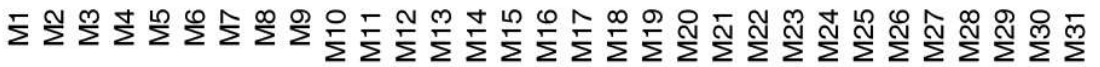

(b) Variable selection results in 2019M12

Figure 6. The variable selection results of the LASSO-MIDAS model when including Baidu Index data and official statistical data. 


\section{Conclusion}

In this study, we examined whether (1) online search engine data are useful for improving the accuracy of tourism demand nowcasting when official statistical data are not available, (2) search engine data are still useful for nowcasting once official statistical data can be used, and (3) if search engine data are useful, when and to what extent such data improve the accuracy of nowcasting. The study also examined whether the LASSO-MIDAS model is effective for nowcasting tourism demand.

Some promising empirical results are obtained from this study. First, compared with the same-frequency OLS-type models, MIDAS-type models do improve the predictive ability when using two data sources that combine daily Baidu index data and monthly official statistical data. The nowcasting results confirm the superiority of MIDAS-type models in analyzing mixed frequency data.

Second, the results obtained by applying Hong Kong's datasets from 2019M1 to 2019M12 show that the nowcasts based on monthly official statistical data are slightly better than those based on the daily Baidu Index data on average. The application of a combination of daily Baidu Index data and monthly official statistical data produces more accurate prediction, when MIDAS-type models are employed.

Third, the nowcasting accuracy of the LASSO-MIDAS model is significantly higher than that of other competing models, which confirms the effectiveness of applying the LASSO-MIDAS model to tourism demand nowcasting. Also, the results of the MCS test further statistically confirm that the LASSO-MIDAS model performs best and is effective in nowcasting tourism demand.

Fifth, the variable selection results of the LASSO-MIDAS model in two cases of only daily Baidu Index data and the combination of the daily updated Baidu Index data and upcoming monthly official statistical data show that the keywords selected in the variable selection process are distributed among six aspects of tourism planning-lodging, dining, attractions, transportation, tours, and shopping. The results show that the web search keywords chosen on the basis of the prior knowledge of the researcher are reasonable. In addition, the available official statistical data also provide useful information for tourism demand nowcasting.

Overall, this study contributes to the extant literature in two ways. First, the study is the first attempt to progressively compare the ability of search engine data, official statistical data, and the combination of the two types of data to nowcast tourism demand. The empirical results confirm that real-time updated search engine data do improve the nowcasting accuracy, while the continuously updated official statistical data should not be ignored either. Therefore, this study provides a useful addition to the existing research on tourism demand nowcasting. The findings suggest that future tourism nowcasting research should take account of a broad range of available, updated information. Second, the study contributes to the tourism forecasting literature by presenting the first attempt to evaluate the applicability and effectiveness of the LASSO-MIDAS model in tourism demand nowcasting. The empirical results confirm that this kind of penalty-based MIDAS model is an effective option when using high-dimensional data. 
There are some directions for future research. First, this study only focuses on Hong Kong as a case destination; more regions and countries can be considered in future research to further generalize the results of this study. Second, other forms of high-dimensional data such as social media data may contain valuable predictive information, and the use of these data in forecasting and nowcasting tourism demand can be explored. Third, this study focuses on short-term nowcasting; future research can further consider whether the effectiveness of different data sources would vary over time in generating longer-term forecasts.

\section{References}

Andreou, E., Ghysels, E. and Kourtellos, A. (2010), "Regression models with mixed sampling frequencies", Journal of Econometrics, Vol. 158 No. 2, pp. 246-261.

Avvenuti, M., Cresci, S., La Polla, M. N., Meletti, C. and Tesconi, M. (2017), "Nowcasting of earthquake consequences using big social data", IEEE Internet Computing, Vol. 21 No. 6, pp. 37-45.

Bai, J. and Ng, S. (2002), "Determining the number of factors in approximate factor models", Econometrica, Vol. 70 No. 1, pp. 191-221.

Baker, S. R., Bloom, N. and Davis, S. J. (2016), "Measuring economic policy uncertainty", Quarterly Journal of Economics, Vol. 131 No. 4, pp. 1593-1636.

Banbura, M., Giannone, D. and Reichlin, L. (2011), "Nowcasting", in Clements, M. P. and Hendry, D. F. (Eds.) Handbook on Economic Forecasting, New York: Oxford University Press.

Bangwayo-Skeete, P. F. and Skeete, R. W. (2015), "Can Google data improve the forecasting performance of tourist arrivals? Mixed-data sampling approach", Tourism Management, Vol. 46, pp. 454-464.

Ceron, A., Curini, L. and Iacus, S. M. (2015), "Using sentiment analysis to monitor electoral campaigns: Method matters - evidence from the United States and Italy", Social Science Computer Review, Vol. 33 No. 1, pp. 3-20.

Chatziantoniou, I., Degiannakis, S., Eeckels, B. and Filis, G. (2016), "Forecasting tourist arrivals using origin country macroeconomics", Applied Economics, Vol. 48 No. 27, pp. 2571-2585.

Chen, J. L., Li, G., Wu, D. C. and Shen, S. (2017), "Forecasting seasonal tourism demand using a multiseries structural time series method", Journal of Travel Research, Vol. 58 No. 1, pp. 92-103.

Dahlhaus, T., Guénette, J. D. and Vasishtha, G. (2017), "Nowcasting BRIC+M in real time", International Journal of Forecasting, Vol. 33 No. 4, pp. 915-935.

Diebold, F. X. and Mariano, R. S. (1995), "Comparing predictive accuracy", Journal of Business and Economic Statistics, Vol. 13 No. 3, pp. 253-263.

Ferrara, L. and Marsilli, C. (2019), "Nowcasting global economic growth: A factor-augmented mixed-frequency approach", The World Economy, Vol. 42 No. 3, pp. 846-875.

Forni, M., Hallin, M., Lippi, M. and Reichlin, L. (2005), "The generalized dynamic factor model", Journal of the American Statistical Association, Vol. 100 No. 471, pp. 
830-840.

Ghysels, E., Santa-Clara, P. and Valkanov, R. (2004), "The MIDAS touch: Mixed data sampling regressions", Mimeo: Chapel Hill, NC.

Ghysels, E., Sinko, A. and Valkanov, R. (2007), "MIDAS regressions: Further results and new directions", Econometric Reviews, Vol. 26 No. 1, pp. 53-90.

Goh, C. (2012), "Exploring impact of climate on tourism demand", Annals of Tourism Research, Vol. 39 No. 4, pp. 1859-1883.

Goh, C., Law, R. and Mok, H. M. K. (2008), "Analyzing and forecasting tourism demand: A rough sets approach", Journal of Travel Research, Vol. 46 No. 3, pp. 327-338.

Gunter, U. and Önder, I. (2016), "Forecasting city arrivals with Google Analytics", Annals of Tourism Research, Vol. 61, pp. 199-212.

Hallin, M. and Liška, R. (2007), "Determining the number of factors in the general dynamic factor model", Journal of the American Statistical Association, Vol. 102 No. 478, pp. 603-617.

Hansen, P. R., Lunde, A. and Nason, J. M. (2011), "The model confidence set", Econometrica, Vol. 79 No. 2, pp. 453-497.

Hyndman, R. J. and Athanasopoulos, G. (2018), Forecasting: Principles and practice, OTexts.

Knotek, E. S., II and Zaman, S. (2017), "Nowcasting U.S. Headline and core inflation", Journal of Money, Credit and Banking, Vol. 49 No. 5, pp. 931-968.

Li, G., Song, H. and Witt, S. F. (2006), "Forecasting tourism demand using econometric models", in Buhalis, D. and Costa, C. (Eds.) Tourism management dynamics, Butterworth-Heinemann, Oxford: Elsevier, pp. 219-228.

Li, G., Wu, D. C., Zhou, M. and Liu, A. (2019), "The combination of interval forecasts in tourism", Annals of Tourism Research, Vol. 75, pp. 363-378.

Li, S., Chen, T., Wang, L. and Ming, C. (2018), "Effective tourist volume forecasting supported by PCA and improved BPNN using Baidu index", Tourism Management, Vol. 68, pp. 116-126.

Li, X. and Law, R. (2019), "Forecasting tourism demand with decomposed search cycles", Journal of Travel Research, Vol. 59 No. 1, pp. 52-68.

Li, X., Law, R., Xie, G. and Wang, S. (2021), "Review of tourism forecasting research with internet data", Tourism Management, Vol. 83, p. 104245.

Li, X., Pan, B., Law, R. and Huang, X. K. (2017), "Forecasting tourism demand with composite search index", Tourism Management, Vol. 59, pp. 57-66.

Liu, H., Liu, Y., Wang, Y. and Pan, C. (2018), "Hot topics and emerging trends in tourism forecasting research: A scientometric review", Tourism Economics, Vol. 25 No. 3, pp. 448-468.

Lourenço, N., Gouveia, C. M. and Rua, A. (2020), "Forecasting tourism with targeted predictors in a data-rich environment", Economic Modelling, doi: 10.1016/j.econmod.2020.03.030.

Ma, Y.-R., Ji, Q. and Pan, J. (2019), "Oil financialisation and volatility forecast: Evidence from multidimensional predictors", Journal of Forecasting, Vol. 38 No. 6, pp. 564-581.

Marsilli, C. (2014), "Variable selection in predictive MIDAS models", Banque de France 
Working Paper.

Nakajima, Y. and Sueishi, N. (2020), "Forecasting the Japanese macroeconomy using high-dimensional data", The Japanese Economic Review, doi: 10.1007/s42973-020-00041-z.

Nesterov, Y. (2005), "Smooth minimization of non-smooth functions", Mathematical Programming, Vol. 103 No. 1, pp. 127-152.

Önder, I. and Gunter, U. (2016), "Forecasting tourism demand with Google Trends for a major European city destination", Tourism Analysis, Vol. 21 No. 2, pp. 203-220.

Pan, B., Wu, D. C. and Song, H. (2012), "Forecasting hotel room demand using search engine data", Journal of Hospitality and Tourism Technology, Vol. 3 No. 3, pp. 196-210.

Rambaccussing, D. and Kwiatkowski, A. (2020), "Forecasting with news sentiment: Evidence with UK newspapers", International Journal of Forecasting, Vol. 36 No. 4, pp. 1501-1516.

Santillana, M., Nguyen, A. T., Dredze, M., Paul, M. J., Nsoesie, E. O. and Brownstein, J. S. (2015), "Combining search, social media, and traditional data sources to improve influenza surveillance", PLoS Computational Biology, Vol. 11 No. 10, pp. e1004513.

Siliverstovs, B. (2017), "Short-term forecasting with mixed-frequency data: A MIDASSO approach", Applied Economics, Vol. 49 No. 13, pp. 1326-1343.

Song, H., Gartner, W. C. and Tasci, A. D. A. (2012), "Visa restrictions and their adverse economic and marketing implications - evidence from China", Tourism Management, Vol. 33 No. 2, pp. 397-412.

Song, H. and Li, G. (2008), "Tourism demand modelling and forecasting - a review of recent research", Tourism Management, Vol. 29 No. 2, pp. 203-220.

Song, H., Lin, S., Witt, S. F. and Zhang, X. (2011), "Impact of financial/economic crisis on demand for hotel rooms in Hong Kong", Tourism Management, Vol. 32 No. 1, pp. 172-186.

Song, H., Qiu, R. T. R. and Park, J. (2019), "A review of research on tourism demand forecasting", Annals of Tourism Research, Vol. 75, pp. 338-362.

Song, H., Witt, S. and Li, G. (2009), The advanced econometrics of tourism demand, London: Routledge.

Song, H., Wong, K. K. F. and Chon, K. K. S. (2003), "Modelling and forecasting the demand for Hong Kong tourism", International Journal of Hospitality Management, Vol. 22 No. 4, pp. 435-451.

Soybilgen, B. and Yazgan, E. (2018), "Evaluating nowcasts of bridge equations with advanced combination schemes for the Turkish unemployment rate", Economic Modelling, Vol. 72, pp. 99-108.

Sun, S. L., Wei, Y. J., Tsui, K. L. and Wang, S. Y. (2019), "Forecasting tourist arrivals with machine learning and internet search index", Tourism Management, Vol. 70, pp. 1-10.

Sun, X., Sun, W., Wang, J., Zhang, Y. and Gao, Y. (2016), "Using a Grey-Markov model optimized by Cuckoo search algorithm to forecast the annual foreign tourist arrivals to China", Tourism Management, Vol. 52, pp. 369-379.

Tibshirani, R. (1996), "Regression shrinkage and selection via the LASSO", Journal of the Royal Statistical Society. Series B (Methodological), Vol. 58 No. 1, pp. 267-288.

Tsaur, R.-C. and Kuo, T.-C. (2011), "The adaptive fuzzy time series model with an application 
to Taiwan's tourism demand", Expert Systems with Applications, Vol. 38 No. 8, pp. 9164-9171.

Uematsu, Y. and Tanaka, S. (2017), "High-dimensional macroeconomic forecasting and variable selection via penalized regression", The Econometrics Journal, Vol. 22 No. 1, pp. 34-56.

Wang, S. and Chaovalitwongse, W. A. (2011), Evaluating and comparing forecasting models, John Wiley \& Sons, Inc.

Wen, L., Liu, C. and Song, H. (2019), "Forecasting tourism demand using search query data: A hybrid modelling approach", Tourism Economics, Vol. 25 No. 3, pp. 309-329.

Wen, L., Liu, C., Song, H. and Liu, H. (2021), "Forecasting tourism demand with an improved mixed data sampling model", Journal of Travel Research, Vol. 60 No. 2, pp. 336-353.

Witt, S. F., Song, H. and Wanhill, S. (2004), "Forecasting tourism-generated employment: The case of Denmark", Tourism Economics, Vol. 10 No. 2, pp. 167-176.

Wu, D. C., Cao, Z., Wen, L. and Song, H. (2020), "Scenario forecasting for global tourism", Journal of Hospitality \& Tourism Research, Vol. 45 No. 1, pp. 28-51.

Wu, D. C., Song, H. and Shen, S. (2017), "New developments in tourism and hotel demand modeling and forecasting", International Journal of Contemporary Hospitality Management, Vol. 29 No. 1, pp. 507-529.

Xu, Q., Zhuo, X., Jiang, C., Liu, X. and Liu, Y. (2018), "Group penalized unrestricted mixed data sampling model with application to forecasting US GDP growth", Economic Modelling, Vol. 75, pp. 221-236.

Yang, Y., Pan, B. and Song, H. (2014), "Predicting hotel demand using destination marketing organization's web traffic data", Journal of Travel Research, Vol. 53 No. 4, pp. 433-447.

Zhang, B., Huang, X., Li, N. and Law, R. (2017), "A novel hybrid model for tourist volume forecasting incorporating search engine data", Asia Pacific Journal of Tourism Research, Vol. 22 No. 3, pp. 245-254.

\section{Author bibliographies}

Han Liu, $\mathrm{PhD}$, is professor in the Center for Quantitative Economics and the Business School at Jilin University.

Ying Liu, is a Ph.D. student in the Business School, Jilin University.

Gang Li, $\mathrm{PhD}$, is Professor of Tourism Economics in the School of Hospitality and Tourism Management at the University of Surrey.

Long Wen, $\mathrm{PhD}$, is assistant professor in the School of Economics at the University of Nottingham Ningbo China. 